\title{
SEROTIPOS DE NEUMOCOCO EN NIÑOS PORTADORES ANTES DE LA VACUNACIÓN ANTINEUMOCÓCICA EN EL PERÚ
}

\author{
Erik H. Mercado ${ }^{1, a}$, Martha Egoavil ${ }^{1, b}$, Sara G. Horna ${ }^{1, c}$, Nancy Torres ${ }^{1, d}$, Ricardo Velásquez ${ }^{1, d}$, \\ María E. Castillo ${ }^{1,2, e}$, Eduardo Chaparro ${ }^{1,3, e}$, Roger Hernández ${ }^{1,3, e}$, Wilda Silva ${ }^{4, e}$, \\ Francisco E. Campos ${ }^{5, \mathrm{e}}$, Andrés Sáenz ${ }^{6, \mathrm{e}}$, Félix Hidalgo ${ }^{7, \mathrm{e}}$, Carolina Letona ${ }^{8, \mathrm{e}}$, Ángel G. Valencia ${ }^{9, \mathrm{e}}$, \\ Rosario Cerpa ${ }^{10, \mathrm{e}}$, Bernardo López-de-Romaña ${ }^{11, \mathrm{e}}$, Jackeline Pando ${ }^{12, \mathrm{e}}$, Berenice Torres ${ }^{1, \mathrm{~d}}$, Fiorella Castillo ${ }^{1, \mathrm{~d}}$, \\ Andrea Calle ${ }^{1, d}$, Synthia Rabanal ${ }^{1, d}$, Theresa J. Ochoa ${ }^{1,13, e}$
}

\begin{abstract}
RESUMEN
Objetivos. Determinar la frecuencia y distribución de serotipos de S. pneumoniae en portadores nasofaríngeos sanos menores de dos años previa al uso universal de la vacuna conjugada antineumocócica en el Perú. Materiales y métodos. Entre los años 2007 y 2009 se tomaron muestras de hisopado nasofaríngeo a 2123 niños sanos entre 2 y 24 meses de edad en los consultorios de crecimiento y desarrollo o vacunación de hospitales y centros de salud de siete ciudades del Perú: costa (Lima, Piura); sierra (Cusco, Abancay, Arequipa y Huancayo) y selva (lquitos). Las cepas de neumococo fueron aisladas e identificadas en el laboratorio central del proyecto en Lima y serotipificadas por reacción de Quellung en el Laboratorio de Referencia de Neumococo del Centro de Control y Prevención de Enfermedades. Resultados. Se encontró $27,0 \%$ (573/2123) de portadores nasofaríngeos sanos de neumococo. En las 526 cepas analizadas se encontraron 42 serotipos; los más frecuentes fueron: 19F (18,1\%), 6B (14,3\%); 23F (8,9\%) y $14(6,5 \%)$. Conclusiones. La distribución de serotipos vacunales en las cepas analizadas fue de $50,0 \%$ para los serotipos presentes en la vacuna conjugada heptavalente; $50,2 \%$ para los serotipos presentes en la vacuna decavalente y $57,2 \%$ para la vacuna $13-v a l e n t e$.
\end{abstract}

Palabras clave: Streptococcus pneumoniae; Portador; Serotipificación; Niño; Perú (fuente: DeCS BIREME).

\section{PNEUMOCOCCAL SEROTYPES IN CARRIER CHILDREN PRIOR TO THE INTRODUCTION OF ANTI-PNEUMOCOCCAL VACCINES IN PERU}

\begin{abstract}
Objectives. To determine the carriage rate and serotype distribution of Streptococcus pneumoniae in the nasopharynx of healthy children younger than 2 years prior to the universal use of the pneumococcal conjugate vaccines in Peru. Materials and methods. Between 2007 and 2009 we collected nasopharyngeal swab samples from 2,123 healthy children aged 2 to 24 months in the vaccination and healthy children consultation offices of pediatric hospitals and health centers in 7 cities in Peru: on the coast (Lima, Piura), highlands (Cusco, Abancay, Arequipa and Huancayo) and amazon basin (Iquitos). The pneumococcal strains were isolated and identified at the central laboratory of the project in Lima, and serotyped by Quellung reaction in the pneumococcal reference laboratory at the Center for Diseases Control and Prevention (CDC). Results. We found $27 \%$ (573/2123) of pneumococcal nasopharyngeal healthy carrier children. Among the 526 analyzed strains, we found 42 serotypes; the most common were: $19 \mathrm{~F}(18.1 \%), 6 \mathrm{~B}(14.3 \%) ; 23 \mathrm{~F}(8.9 \%)$ and $14(6.5 \%)$. Conclusions. The distribution of vaccine serotypes in the analyzed strains was of $50 \%$ for the serotypes present in the seven-valent vaccine, $50.2 \%$ for the serotypes present in the ten-valent vaccine and $57.2 \%$ for those present in the thirteen-valent vaccine.
\end{abstract}

Key words: Streptococcus pneumoniae; Carriage; Serotyping; Child; Peru (source: MeSH NLM).

\footnotetext{
Facultad de Medicina Alberto Hurtado, Universidad Peruana Cayetano Heredia. Lima, Perú.

Instituto Nacional de Salud del Niño. Lima, Perú.

Hospital Nacional Cayetano Heredia. Lima, Perú.

4 Hospital Nacional Edgardo Rebagliati Martins. Lima, Perú.

5 Hospital Nacional Docente Madre-Niño San Bartolomé. Lima, Perú.

6 Hospital Nacional Daniel A. Carrión. Lima, Perú.

Hospital Regional del Cusco. Cusco, Perú.

8 Centro de Salud de Wanchaq. Cusco, Perú.

9 Hospital Guillermo Díaz de la Vega, Abancay. Apurímac, Perú.

${ }^{10}$ Hospital EsSalud de Yanahuara. Arequipa, Perú.

${ }^{11}$ Policlínico Metropolitano de Arequipa. Arequipa, Perú.

${ }_{12}$ Clinical Lecturer, Department of Paediatrics and Child Health, University College Cork. Irlanda.

${ }^{13}$ University of Texas School of Public Health. Texas, Estados Unidos de Norteamérica.

a Biólogo; ${ }^{\mathrm{b}}$ médico cirujano; ${ }^{\mathrm{c}}$ tecnóloga médico; ${ }^{\mathrm{d}}$ estudiante de Medicina; ${ }^{\mathrm{e}}$ médico infectólogo pediatra
}

Recibido: 04-11-11 Aprobado: 22-02-12 


\section{INTRODUCCIÓN}

Las infecciones por Streptococcus pneumoniae continúa siendo una de las causas más frecuentes de enfermedad invasiva bacteriana, particularmente en niños y ancianos ${ }^{(1)}$. En el Perú, en el caso específico de los niños, el S. pneumoniae es aislado con alta frecuencia en neumonía y meningoencefalitis bacteriana aguda ${ }^{(2-4)}$. En la actualidad se conocen más de 90 serotipos de neumococo con potencial para producir enfermedades en el ser humano. Se ha demostrado ampliamente que la colonización de la nasofaringe por $S$. pneumoniae es mayor durante los primeros dos años de vida ${ }^{(5)}$. Esta colonización es importante no solo porque es la condición inicial para desarrollar una enfermedad neumocócica invasiva, sino también porque proporciona la base para la expansión horizontal del neumococo en la población ${ }^{(6)}$. Se estima que la colonización puede ser más o menos duradera en función a diferentes factores, tales como la capacidad invasiva de cada serotipo, presión antibiótica y la vacunación ${ }^{(7)}$.

La incorporación de la vacuna antineumocócica conjugada heptavalente (PCV7) en los programas nacionales de vacunación reduce la incidencia de enfermedad neumocócica invasiva, neumonía, otitis media y enfermedad neumocócica asociada a resistencia antibiótica en niños y adultos. Sin embargo, puede llevar a un incremento gradual en la proporción de los serotipos que no estaban presentes en las vacunas ${ }^{(8)}$, además de reducir la portación nasofaríngea con serotipos vacunales ${ }^{(9)}$.

En el Perú existen escasas investigaciones sobre la distribución de serotipos en portadores nasofaríngeos ${ }^{(10,11)}$. Estos estudios son antiguos y se han centrado únicamente en niños de la ciudad de Lima. Basados en lo anterior y con la finalidad de poder estimar la frecuencia y distribución basal de serotipos de $S$. pneumoniae previa a la introducción de la vacuna PCV7 en nuestro país, realizamos este estudio en portadores nasofaríngeos sanos menores de dos años de edad en siete ciudades del Perú.

\section{MATERIALES Y MÉTODOS}

\section{POBLACIÓN}

Este fue un estudio multicéntrico transversal realizado entre los años 2007 y 2009. Los criterios de inclusión fueron niños sanos entre 2 y 24 meses que acudieron a sus controles de niño sano en los consultorios de crecimiento y desarrollo o vacunación, de forma ambulatoria en los hospitales o centros de salud. Se excluyeron del estudio a niños con enfermedad

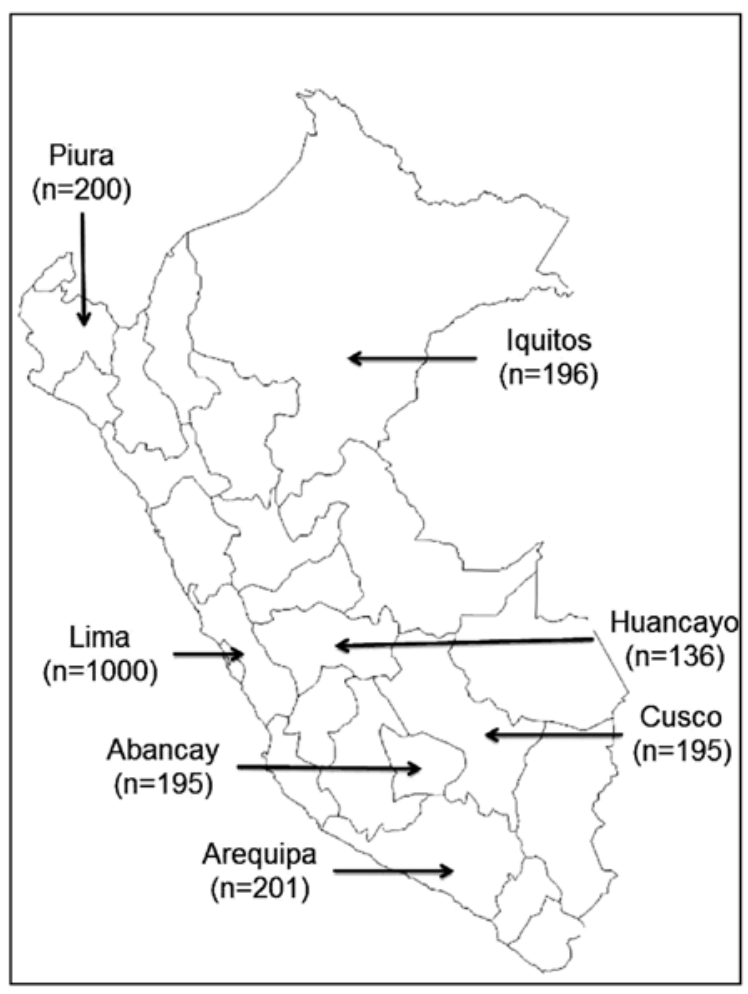

Figura 1. Ciudades del Perú en el que se realizó el estudio de portadores nasofaríngeos de S. pneumoniae 2007-2009. $\mathrm{n}$ : número de niños incluidos en cada ciudad.

grave que requerían hospitalización o transferencia a Emergencia.

Los niños fueron enrolados en siete ciudades en las tres regiones del Perú: costa (Lima, Piura); sierra (Cusco, Abancay, Arequipa y Huancayo) y selva (Iquitos) (Figura 1, Tabla 1). El tamaño de la muestra se determinó por separado para Lima y para el interior del país. Se estimó que el $20 \%$ de las cepas encontradas fueran multirresistentes, por lo que se necesitarían aproximadamente 250 cepas para Lima y 250 para el resto. Se estimó un porcentaje de portador aproximado de $25 \%$, requiriéndose muestrear aproximadamente 1000 niños en la ciudad de Lima y 1000 en las ciudades del interior del país.

El enrolamiento se realizó por conveniencia, de manera secuencial, empezando en Lima, seguido de Arequipa, Abancay, Piura, Cusco, Huancayo e Iquitos; ello tomó, en promedio, entre dos y seis meses por cada hospital, hasta completar el número de niños por ciudad. Por lo tanto, hubo diferencias estacionales en el enrolamiento en cada ciudad. La muestra estuvo compuesta por aproximadamente el mismo número de niños en los siguientes grupos de edad: 2 a 6 meses, 7 a 12 meses, 
Tabla 1. Porcentaje de portador nasofaríngeo de $S$. pneumoniae en cada ciudad, hospital y centro de salud (CS) participante.

\begin{tabular}{|c|c|c|}
\hline Ciudad & \multirow{2}{*}{$\begin{array}{c}\text { Número } \\
\text { de niños } \\
\text { muestreados }\end{array}$} & \multirow{2}{*}{$\begin{array}{c}\text { Número } \\
\text { de niños } \\
\text { portadores } \\
(\%)\end{array}$} \\
\hline $\begin{array}{l}\text { Hospital / centro de } \\
\text { salud }\end{array}$ & & \\
\hline \multicolumn{3}{|l|}{ COSTA } \\
\hline Lima & 1000 & $311(31,1)$ \\
\hline $\begin{array}{l}\text { Instituto Nacional } \\
\text { de Salud del Niño }\end{array}$ & 200 & $61(30,5)$ \\
\hline $\begin{array}{l}\text { Hospital Edgardo } \\
\text { Rebagliati Martins }\end{array}$ & 200 & $53(26,5)$ \\
\hline $\begin{array}{l}\text { Hospital Madre-Niño } \\
\text { San Bartolomé }\end{array}$ & 200 & $60(30,0)$ \\
\hline $\begin{array}{l}\text { Hospital Nacional } \\
\text { Cayetano Heredia }\end{array}$ & 200 & $60(30,0)$ \\
\hline $\begin{array}{l}\text { Hospital Nacional } \\
\text { Daniel A. Carrión }\end{array}$ & 200 & $77(38,5)$ \\
\hline Piura & 200 & $48(24,0)$ \\
\hline CLAS Catacaos & 78 & $18(23,1)$ \\
\hline EsSalud - Sullana & 56 & $10(17,9)$ \\
\hline Hospital Santa Rosa & 38 & $17(44,7)$ \\
\hline $\begin{array}{l}\text { Hospital Regional } \\
\text { Cayetano Heredia }\end{array}$ & 28 & $3(10,7)$ \\
\hline \multicolumn{3}{|l|}{ SIERRA } \\
\hline Cusco & 195 & $47(24,1)$ \\
\hline Hospital EsSalud Cusco & 49 & $12(24,5)$ \\
\hline CS Wanchaq & 47 & $10(21,3)$ \\
\hline $\begin{array}{l}\text { Hospital Regional del } \\
\text { Cusco }\end{array}$ & 49 & $11(22,4)$ \\
\hline CS Urubamba & 50 & $14(28,0)$ \\
\hline Abancay & 195 & $41(21,0)$ \\
\hline $\begin{array}{l}\text { Hospital Guillermo Díaz de } \\
\text { la Vega }\end{array}$ & 195 & $41(21,0)$ \\
\hline Arequipa & 201 & $34(16,9)$ \\
\hline $\begin{array}{l}\text { Hospital III de Yanahuara - } \\
\text { EsSalud }\end{array}$ & 132 & $15(11,4)$ \\
\hline $\begin{array}{l}\text { Policlínico Metropolitano } \\
\text { de Arequipa }\end{array}$ & 69 & $19(27,5)$ \\
\hline Huancayo & 136 & $30(22,1)$ \\
\hline Hospital El Carmen & 136 & $30(22,1)$ \\
\hline \multicolumn{3}{|l|}{ SELVA } \\
\hline Iquitos & 196 & $62(31,6)$ \\
\hline EsSalud de lquitos & 148 & $53(35,8)$ \\
\hline Hospital Regional lquitos & 26 & $8(30,8)$ \\
\hline Hospital de apoyo & 22 & $1(4,5)$ \\
\hline Total & 2123 & $573(27,0)$ \\
\hline
\end{tabular}

13 a 18 meses y 19 a 24 meses. En ninguna de estas ciudades se había iniciado la vacunación (PCV7) en la región, antes o durante la ejecución del estudio.

Este estudio fue aprobado por el Comité Institucional de Ética de la Universidad Peruana Cayetano Heredia y de cada una de las instituciones participantes.

\section{PROCEDIMIENTOS DEL ESTUDIO}

Previa a la toma de muestras, se obtuvo el consentimiento informado de los padres y se llenó una ficha con los datos del niño. Se tomaron muestras de hisopado nasofaríngeo a los niños usando un hisopo delgado de alginato de calcio con vástago de aluminio (Fisher, Healthcare, Pittsburgh, PA). Dichas muestras fueron tomadas por personal entrenado (pediatras o enfermeras). Se empleó como medio de transporte el medio Amies con carbón activado (Copan Innovation, Brescia, Italia), excepto en las ciudades de Piura, donde se empleó leche descremada triptona glucosa glicerol (LTGG) y en la ciudad de Lima, donde las muestras fueron sembradas directamente en placas de agar sangre, además de ser transportadas en caldo TSB (trypticase soy broth).

En todos los casos, las muestras fueron transportadas a temperatura ambiente al laboratorio central del proyecto en Lima (Laboratorio de Microbiología del Instituto de Medicina Tropical Alexander von Humboldt) en un lapso no mayor a una semana para las muestras colectadas en el interior del país y el mismo día para las muestras colectadas en Lima.

\section{IDENTIFICACIÓN DE S. pneumoniae}

En el laboratorio central del proyecto, las muestras se sembraron en agar sangre (TSB suplementado con $5 \%$ de sangre de carnero). Se determinó el aislamiento como S. pneumoniae en base a la morfología de la colonia, alfa hemólisis, coloración Gram, solubilidad en bilis y sensibilidad a la optoquina ${ }^{(12)}$. Las cepas identificadas como S. pneumoniae se guardaron a $-70^{\circ} \mathrm{C}$ hasta su posterior envío a un laboratorio de referencia en un medio de transporte previamente validado silica packs ${ }^{(13)}$.

\section{SEROTIPIFICACIÓN DE S. pneumoniae}

La determinación de serotipos de S. pneumoniae se realizó en el Laboratorio de Neumococo del Centro de Control y Prevención de Enfermedades (CDC) (Streptococcus Laboratory, Center for Disease Control and Prevention, Atlanta, Estados Unidos de Norteamérica). La serotipificación se realizó por el método de hinchamiento capsular (reacción de Quellung), empleando el esquema y los antisueros producidos en el CDC.

\section{ANÁLISIS ESTADÍSTICO}

Los cálculos de frecuencia y comparación entre poblaciones portadoras y no portadoras se realizó a través de la prueba de Chi cuadrado con el programa Epi Info v.3.5. Se consideró un $p<0,05$ como significativo. 


\section{RESULTADOS}

\section{PORCENTAJE DE PORTADORES}

Se enrolaron 2123 niños; 1145 niños entre 2 - 12 meses (edad promedio $6,7 \pm 3,4 \mathrm{~m}$ ) y 978 niños entre $13-24$ meses (edad promedio $18,3 \pm 3,5 \mathrm{~m}$ ). El $52,7 \%$ de los niños enrolados fueron varones. La totalidad de los niños no habían recibido ninguna vacuna antineumocócica antes del hisopado nasofaríngeo. El porcentaje global de portador fue de $27 \%$ con un rango de variación entre 5 y $45 \%$ en los diferentes hospitales o centros de salud. Se encontró un mayor porcentaje de portadores en las ciudades de Lima e Iquitos (Tabla 1).

Al analizar el porcentaje de portadores por grupo etario, encontramos que fue mayor en los niños entre 7 y 12 meses de edad ( $31 \%$ ), en comparación con los niños entre 2 y 6 meses de edad $(23 \%)(p<0,01)$. Por otro lado, encontramos que el $30 \%$ de los niños muestreados con diagnóstico de rinorrea estuvieron colonizados por neumococo, porcentaje relativamente mayor al observado en los niños considerados como sanos al momento del muestreo $(23 \%)$.
Al estudiar algunos factores asociados con la condición de portador, no se encontraron diferencias significativas con los no portadores, en cuanto al uso de antibióticos en los tres meses previos (36 y $37 \%$ ), la asistencia a guarderías (4 y $5 \%$ ) y la presencia de más de siete personas en el hogar (19 y 18\%).

El $91,8 \%(526 / 573)$ de las cepas aisladas fueron recuperadas para determinar el serotipo. El resto de las cepas se perdió durante el almacenamiento o transporte.

\section{DISTRIBUCIÓN DE SEROTIPOS DE S. pneumoniae}

De las 526 cepas de S. pneumoniae estudiadas 275 fueron aisladas en niños de Lima y 251 en provincias. Se encontraron 42 serotipos de los más de 90 buscados. Los serotipos más frecuentes fueron: 19F, 6B, 23F, 14, $6 \mathrm{~A}, 11 \mathrm{~A}, 6 \mathrm{C}, 19 \mathrm{~A}$ y $23 \mathrm{~A}$ (Tabla 2). Los tres serotipos más frecuentes encontrados en portadores nasofaríngeos sanos por ciudad fueron: en Lima 19F $(20 \%), 6 B(12 \%)$ y 23F (9\%); en lquitos $23 \mathrm{~F}(16 \%), 19 \mathrm{~F}(11 \%)$ y $6 \mathrm{~B}(11 \%)$; en Cusco $14(13 \%), 19 \mathrm{~F}(11 \%)$ y $6 \mathrm{~B}(11 \%)$; en Piura $6 \mathrm{~B}$ (26\%), $19 \mathrm{~F}(13 \%)$ y 14 (9\%); en Abancay 6B (24\%), 19F

TABLA 2. Distribución de serotipos de $S$. pneumoniae por región y frecuencia global de serotipos aislados en niños portadores entre 2 a 24 meses de edad.

\begin{tabular}{|c|c|c|c|c|c|c|c|c|}
\hline Serotipo & $\begin{array}{c}\text { Lima } \\
n=275\end{array}$ & $\begin{array}{c}\text { Iquitos } \\
n=57\end{array}$ & $\begin{array}{c}\text { Cusco } \\
n=47\end{array}$ & $\begin{array}{l}\text { Piura } \\
n=46\end{array}$ & $\begin{array}{c}\text { Abancay } \\
n=41\end{array}$ & $\begin{array}{c}\text { Arequipa } \\
n=30\end{array}$ & $\begin{array}{c}\text { Huancayo } \\
n=30\end{array}$ & $\begin{array}{c}\text { Frecuencia total } \\
n=526, n(\%)\end{array}$ \\
\hline $19 \mathrm{~F}$ & 56 & 6 & 5 & 6 & 8 & 6 & 8 & $95(18,1)$ \\
\hline $6 \mathrm{~B}$ & 32 & 6 & 5 & 12 & 10 & 4 & 6 & $75(14,3)$ \\
\hline 14 & 13 & 2 & 6 & 4 & 4 & 4 & 1 & $34(6,5)$ \\
\hline $6 \mathrm{~A}$ & 9 & 5 & 2 & 3 & 2 & 0 & 1 & $22(4,2)$ \\
\hline $11 \mathrm{~A}$ & 12 & 0 & 2 & 1 & 2 & 1 & 1 & $19(3,6)$ \\
\hline $6 \mathrm{C}$ & 4 & 6 & 1 & 3 & 0 & 2 & 2 & $18(3,4)$ \\
\hline $23 \mathrm{~A}$ & 12 & 0 & 2 & 0 & 1 & 0 & 0 & $15(2,9)$ \\
\hline $15 \mathrm{~A}$ & 10 & 1 & 0 & 1 & 0 & 0 & 0 & $12(2,3)$ \\
\hline $23 B$ & 3 & 4 & 1 & 1 & 0 & 2 & 0 & $11(2,1)$ \\
\hline $35 \mathrm{~F}$ & 5 & 1 & 3 & 0 & 1 & 0 & 1 & $11(2,1)$ \\
\hline $9 \mathrm{~V}$ & 3 & 0 & 3 & 1 & 2 & 0 & 1 & $10(1,9)$ \\
\hline $15 \mathrm{C}$ & 7 & 0 & 0 & 2 & 1 & 0 & 0 & $10(1,9)$ \\
\hline $15 B$ & 6 & 0 & 1 & 0 & 0 & 0 & 2 & $9(1,7)$ \\
\hline 13 & 3 & 1 & 2 & 1 & 0 & 0 & 1 & $8(1,5)$ \\
\hline 34 & 3 & 1 & 2 & 1 & 0 & 0 & 0 & $7(1,3)$ \\
\hline $24 \mathrm{~F}$ & 5 & 0 & 0 & 0 & 0 & 0 & 0 & $5(1,0)$ \\
\hline $33 \mathrm{~F}$ & 3 & 0 & 0 & 0 & 1 & 1 & 0 & $5(1,0)$ \\
\hline $7 \mathrm{C}$ & 0 & 1 & 0 & 1 & 0 & 0 & 2 & $4(0,8)$ \\
\hline $16 \mathrm{~F}$ & 2 & 2 & 0 & 0 & 0 & 0 & 0 & $4(0,8)$ \\
\hline $22 \mathrm{~F}$ & 1 & 1 & 1 & 0 & 0 & 1 & 0 & $4(0,8)$ \\
\hline 21 & 1 & 0 & 0 & 1 & 1 & 0 & 0 & $3(0,6)$ \\
\hline 20 & 1 & 0 & 1 & 0 & 1 & 0 & 0 & $3(0,6)$ \\
\hline 35B & 1 & 0 & 1 & 0 & 1 & 0 & 0 & $3(0,6)$ \\
\hline 4 & 2 & 0 & 0 & 0 & 0 & 0 & 0 & $2(0,4)$ \\
\hline $6 \mathrm{D}$ & 0 & 0 & 1 & 0 & 0 & 1 & 0 & $2(0,4)$ \\
\hline $9 \mathrm{~A}$ & 1 & 0 & 0 & 0 & 0 & 1 & 0 & $2(0,4)$ \\
\hline $10 \mathrm{~F}$ & 0 & 2 & 0 & 0 & 0 & 0 & 0 & $2(0,4)$ \\
\hline $17 \mathrm{~F}$ & 0 & 0 & 2 & 0 & 0 & 0 & 0 & $2(0,4)$ \\
\hline $18 \mathrm{~A}$ & 0 & 1 & 0 & 0 & 0 & 1 & 0 & $2(0,4)$ \\
\hline $18 \mathrm{~B}$ & 2 & 0 & 0 & 0 & 0 & 0 & 0 & $2(0,4)$ \\
\hline $35 \mathrm{~A}$ & 1 & 0 & 0 & 0 & 0 & 0 & 1 & $2(0,4)$ \\
\hline
\end{tabular}

Otros: serotipos 7F, 9N, 10B, 11D, 19C, 28A, 33B/D, 39 


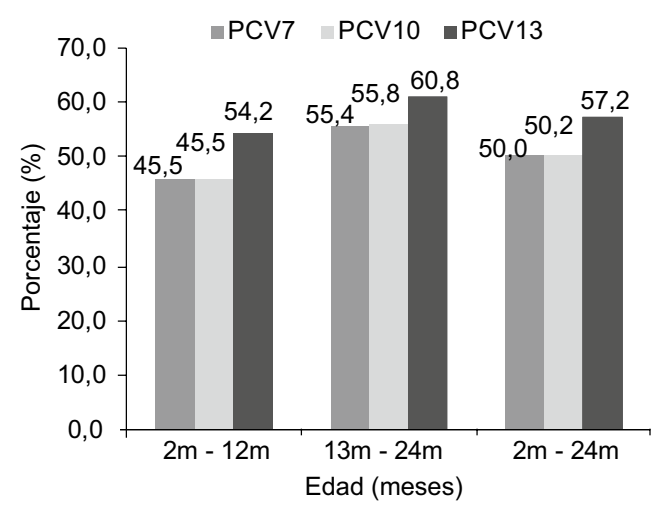

Figura 2. Distribución de serotipos vacunales de S. pneumoniae en portadores previa a la introducción universal de la vacuna PCV7 en el Perú*. Niños de $2 m-12 m$ ( $n=286)$; niños de $13 m-$ $24 \mathrm{~m}(\mathrm{n}=240)$; total $(n=526)$.

* Serotipos incluidos en la vacuna conjugada heptavalente (PCV7): 4, $6 \mathrm{~B}, 9 \mathrm{~V}, 14,18 \mathrm{C}, 19 \mathrm{~F}, 23 \mathrm{~F}$; serotipos incluidos en la vacuna conjugada decavalente (PCV10): serotipos de la PCV7 más 1, 5, 7F; y serotipos incluidos en la vacuna conjugada 13-valente (PCV13): serotipos de la PCV10 más $3,6 \mathrm{~A}, 19 \mathrm{~A}$.

(20\%) y $14(10 \%)$; en Arequipa $19 \mathrm{~F}(20 \%), 23 \mathrm{~F}(17 \%)$ y $6 \mathrm{~B}(13 \%)$ y $14(13 \%)$ y en Huancayo $19 \mathrm{~F}(20 \%), 6 \mathrm{~B}$ $(20 \%)$ y $23 \mathrm{~F}(7 \%)$. Cabe resaltar el hallazgo de un nuevo serotipo $6 \mathrm{D}$, en dos niños procedentes de la sierra de Arequipa y Cusco.

\section{SEROTIPOS VACUNALES}

La distribución de serotipos vacunales en las cepas estudiadas fue de $50 \%$ para los serotipos presentes tanto en la vacuna conjugada heptavalente PCV7, como en la vacuna decavalente PCV10 y ligeramente mayor, $57 \%$, cuando evaluamos los serotipos incluidos en la vacuna 13-valente PCV13 (Figura 2). Los serotipos vacunales $1,3,5$, y $18 \mathrm{C}$ no estuvieron presentes en las cepas analizadas. El $54,4 \%$ de las cepas analizadas fueron aisladas de niños entre 2 y 12 meses y el resto de cepas a partir de niños entre 13 y 24 meses. La distribución de serotipos de $S$. pneumoniae fue similar en ambos grupos de edad en todas las ciudades estudiadas. Los serotipos no vacunales conformaron un $34,2 \%$ de las cepas analizadas: $39 \%$ en niños entre 2 y 12 meses y $29 \%$ en niños entre 13 y 24 meses; mientras que las cepas no tipificables conformaron un $8,6 \%: 7 \%$ en niños entre 2 y 12 meses y $10 \%$ en niños entre 13 y 24 meses.

\section{DISCUSIÓN}

En el presente estudio se encontró $27 \%$ de portadores nasofaríngeos de neumococo en niños entre 2 y 24 meses de edad que acudieron a su control de rutina en los diferentes hospitales y centros de salud de las siete ciudades que participaron en el estudio. Este porcentaje de portadores es muy similar a lo encontrado en Venezuela ${ }^{(14)}$ y México ${ }^{(15)}$, pero menor al $41-45 \%$ que se reportó previamente en un estudio realizado en Lima (11) y casi el doble a los valores encontrados en Uruguay ${ }^{(16)}$.

Las diferencias encontradas en los porcentajes de portación de neumococo en las diferentes ciudades del Perú puede deberse a múltiples factores. En primer lugar, la edad y condiciones asociadas al estado de portador (estado de salud, hacinamiento, asistencia a guarderías, uso previo de antibióticos, etc.) puede haber sido distinto en las diferentes poblaciones estudiadas. Sin embargo, no fue objetivo de este estudio determinar los factores asociados a colonización en las diversas regiones. No obstante, cabe señalar que desde el punto de visto metodológico, sí hubo diferencias en los métodos de conservación y transporte de la muestra, lo que sí podría afectar la probabilidad de recuperación de las cepas de neumococo. En tal sentido, en los hospitales de Lima, por facilidades logísticas, las muestras de hisopado se sembraron en placa directamente $\mathrm{y}$, además, se transportaron y cultivaron el mismo día en el laboratorio central del estudio. Por el contrario, en el resto de establecimientos, las muestras fueron enviadas semanalmente al laboratorio central y se usaron métodos diferentes para su transporte. En tal sentido, era de esperar por ejemplo que las muestras tomadas en la ciudad de Piura y transportadas en LTGG, tal como lo recomienda la Organización Mundial de la Salud, presentaran mayor porcentaje de positividad, sin embargo no fue así. No tenemos argumentos sólidos para explicar estas diferencias.

El $91 \%$ de las cepas analizadas fueron serotipificables, similar a lo descrito por Quiroga et al. en Venezuela ${ }^{(14)}$. Los serogrupos más frecuentemente aislados en nuestro estudio fueron $6(22 \%), 19(21 \%), 23(14 \%)$, y $14(6 \%)$, similar a lo encontrado en estudios previos en niños portadores nasofaríngeos en Lima ${ }^{(10,11)}$. Los serotipos más frecuentes encontrados fueron $19 \mathrm{~F}, 6 \mathrm{~B}, 23 \mathrm{~F}, 14,6 \mathrm{~A}$, $11 \mathrm{~A}, 6 \mathrm{C}, 19 \mathrm{~A}$ y $23 \mathrm{~A}$, correspondiendo al $65 \%$ (340/526) del total de las cepas analizadas, similar a lo encontrado en estudios previos realizados en portadores en Chile y México (15,17).

Recientemente las cepas de S. pneumoniae que pertenecen al serotipo $6 \mathrm{D}$ han sido aisladas en infecciones invasivas y consideradas como cepas potencialmente virulentas ${ }^{(18)}$. Su aislamiento en portadores nasofaríngeos se ha asociado a una presión de selección de la vacuna antineumocócica heptavalente (PCV7) ${ }^{(19)}$. Sin embargo, este es el primer hallazgo en Latinoamérica 
del serotipo 6D, aislado en niños de Cusco y Arequipa ${ }^{(20)}$, indicando que puede también aislarse en población infantil en lugares donde aún no se ha introducido la vacuna antineumocócica.

Se ha demostrado claramente que la vacunación con PCV7 previene las enfermedades causadas por los serotipos incluidos en la vacuna. En nuestro estudio, la cobertura teórica de la vacuna PCV7 fue del $50 \%$, similar a lo reportado recientemente en Bolívar, Venezuela (21). Llama la atención que no se encontraron niños colonizados con los serotipos 1 y 5 , serotipos importantes en Latinoamérica asociados con enfermedad neumocócica invasiva ${ }^{(22,23)}$, tal como lo demuestra un estudio en Chile ${ }^{(22)}$. Solo un niño estuvo colonizado con el serotipo 7F; por lo que no se encontró mayor beneficio al evaluar la cobertura teórica de la vacuna conjugada PCV10 (50,2\%). Sin embargo, este porcentaje aumentó a $57,2 \%$ cuando se consideran los serotipos de la vacuna PCV13, principalmente debido a la presencia de los serotipos $6 \mathrm{~A}$ y $19 \mathrm{~A}$.

Es importante considerar que del total de cepas analizadas, el $3 \%$ perteneció al serotipo $19 \mathrm{~A}$, en ausencia de vacunación neumocócica. En los países donde se introdujo la vacuna PCV7, además de haberse removido los serotipos vacunales, entre los serotipos emergentes asociados con enfermedad neumocócica invasiva, se encuentra el serotipo $19 \mathrm{~A}^{(24)}$. Consideramos que la introducción de la vacuna PCV7 en nuestro país es importante para reducir la tasa de mortalidad; sin embargo, hay que tener en cuenta que serotipos no incluidos en esta vacuna, se encuentran circulando en la comunidad y podrían cobrar importancia.

Llama también la atención la baja prevalencia del serotipo 14, el cual es el principal serotipo asociado con enfermedad invasiva en nuestro medio ${ }^{(4,25)}$. En un estudio similar, en 211 cepas aisladas de niños portadores en Lima entre los años 1997 y 2003, tampoco se encontraron los serotipos 1,5 y $7 \mathrm{~F}{ }^{(11)}$. No obstante, hay que tomar estos resultados con mucho cuidado, dado que la distribución de serotipos en portadores nasofaríngeos solo nos da una idea de los serotipos circulantes en una determinada población, en un determinado momento, pero no necesariamente representan los mismos serotipos ni la misma frecuencia de los serotipos que se encontrarían en enfermedades invasivas (bacteriemia, meningitis).

Este estudio presenta las siguientes limitaciones: en primer lugar, el tiempo de envío de las muestras de provincia al laboratorio central en Lima no fue óptimo en todos los casos y se emplearon medios de transporte diferentes para el envío de las muestras al laboratorio central del proyecto; lo que pudo influir en una menor recuperación de las cepas; es decir, el porcentaje de portador podría haber sido mayor. En segundo lugar, tuvimos un $9 \%$ del total de las cepas aisladas que se perdieron, ya sea por dificultad en el almacenamiento (no crecieron en la resiembra) o se contaminaron durante el transporte del laboratorio central a la CDC. En tercer lugar, las ciudades no fueron seleccionadas en forma aleatoria sino por conveniencia, al igual que la selección de los niños dentro de cada establecimiento; por lo tanto, las conclusiones son válidas para las ciudades estudiadas y no pretendemos extrapolar conclusiones para todo el país. Sin embargo, pese a todas estas limitaciones, consideramos que el número total de cepas aisladas y recuperadas para serotipificación (526) conforman una muestra significativa y representativa para los fines del estudio, considerando que este estudio es el primero realizado fuera del ámbito de la ciudad de Lima y trató de incluir algunas ciudades de las tres regiones del país.

En nuestro país la vacuna conjugada heptavalente antineumocócica (PCV7) se introdujo en el Calendario Nacional de Vacunación, para todos los niños menores de dos años, desde julio del año 2009; sin embargo, las coberturas de vacunación fueron muy bajas en los primeros meses. El presente estudio, corresponde a nuestro entender, el estudio con mayor cantidad de cepas de neumococo procedentes de varias regiones del país, lo que representa información muy importante para conocer los serotipos circulantes basales antes de la introducción de las vacunas conjugadas en el calendario de vacunación nacional. Dado que los estudios con cepas invasivas son también muy limitados en nuestro país, se recomienda hacer un estudio similar después de la introducción de la vacuna antineumocócica, luego de alcanzar coberturas vacunales altas y sostenidas, para poder estudiar los cambios en los serotipos circulantes y poder medir el impacto de la vacunación en el estado de portador.

\section{AGRADECIMIENTOS}

A los doctores Bernard Beall, Lesley McGee y Keith Klugman del Laboratorio de Referencia en Neumococo de la CDC (Streptococcus Laboratory, Center for Disease Control and Prevention, Atlanta, Estados Unidos) por la serotipificacion de las cepas. Agradecemos a las siguientes personas que colaboraron en la ejecución del estudio: Humberto Guerra, Lida Mejía, Benita Larota y Dora Leandro (Instituto de Medicina Tropical Alexander von Humboldt, Universidad Peruana Cayetano Heredia, Lima); James Solís, Esperanza Ticona y Rosminy Ayquipa (Hospital Guillermo Díaz de la Vega, Abancay); Felicitas Hanco, Diomar Aedo y Elizabeth Lacroix (Hospital Essalud Cusco, Centro de Salud Wanchaq y Centro de Salud Urubamba, Cusco); Luis Porras, Andrés Barriga, Fredy Camayo y Jenny Arteaga (Hospital El Carmen y Hospital Daniel Alcides Carrión, Huancayo); Miguel Mestanza y Jorge 
Díaz (Hospital Regional y Hospital EsSalud, Iquitos); Jorge Frías, Giovanna Wong, Oscar Requena y Víctor Velarde (CLAS Catacaos, Hospital de EsSalud de Sullana, Hospital Santa Rosa y Hospital Regional Cayetano Heredia, Piura).

\section{Contribuciones de autoría}

EHM, ME, MEC, EC, RE, WS y TJO participaron en la concepción y diseño del trabajo. ME, SGH, MEC, EC, RH, WS, $\mathrm{FEC}, \mathrm{AS}, \mathrm{FH}, \mathrm{CL}, \mathrm{AV}, \mathrm{RC}$ y BLR participaron en la recolección/ obtención de resultados y aporte de pacientes al estudio. EHM, NT, RV, JP, BT, FC, AC, SR y TJO participaron en la redacción del manuscrito, EHM, SH y TJO participaron en el análisis e interpretación de datos. Adicionalmente EHM y TJO participaron en la recolección /obtención de resultados. TJO obtuvo el financiamiento. Todos los autores revisaron en forma crítica versiones preliminares del manuscrito y aprobaron la versión final del trabajo.

\section{Fuentes de financiamiento}

Este trabajo fue financiado por el fondo concursable de la Facultad de Medicina Alberto Hurtado de la Universidad Peruana Cayetano Heredia, otorgado a la Dra. Theresa Ochoa.

\section{Conflictos de interés}

TJO, EC, SGH, WS, RE, MEC, ME, AS y FEC han recibido financiamiento por el Laboratorio Wyeth (actualmente Pfizer) para el estudio de cepas invasivas de $S$. pneumoniae. EHM recibió entrenamiento en serotipificación de neumococo en el CDC a través del proyecto "Global Strain Bank Project" financiado por PATH (Partnership Action on Tobacco and Health).

\section{REFERENCIAS BIBLIOGRÁFICAS}

1. Valenzuela MT, O'Loughlin R, De La Hoz F, Gomez E, Constenla D, Sinha A, et al. The burden of pneumococcal disease among Latin American and Caribbean children: review of the evidence. Rev Panam Salud Publica. 2009;25(3):270-9.

2. Padilla J, Lindo F, Rojas R, Tantaleán J, Suárez V, Cabezas C, et al. Etiology of community acquired pneumonia in children 2-59 months old in two ecologically different communities from Peru. Arch Argent Pediatr. 2010;108(6):516-23.

3. Vizcarra NO. Meningitis supurada: Estudio epidemiológico, clínico y terapeútico de 319 casos [Tesis de Bachiller]. Lima: Facultad de Medicina, Universidad Peruana Cayetano Heredia; 1983.

4. Ochoa TJ, Egoavil M, Castillo ME, Reyes I, Chaparro E, Silva W, et al. Invasive pneumococcal diseases among hospitalized children in Lima, Peru. Rev Panam Salud Publica. 2010;28(2):121-127

5. Lloyd-Evans NT, O'Dempsey TJ, Baldeh I, Secka O, Demba E, Todd JE, et al. Nasopharyngeal carriage of pneumococci in Gambian children and their families. Pediatr Infect Dis J. 1996;15(10):866-71.

6. Bogaert D, De Groot R, Hermans PW. Streptococcus pneumoniae colonisation: the key to pneumococcal disease. Lancet Infect Dis. 2004;4(3):144-54.

7. García-Vera C. Estado de portador de neumococo en niños y su relación con la enfermedad invasiva: ¿qué ha cambiado tras la introducción de la vacuna conjugada?. Revista Pediatría de Atención Primaria. 2010;12(47):457-82.

8. Frazão N, Brito-Avô A, Simas C, Saldanha J, Mato R, Nunes $S$, et al. Effect of the seven-valent conjugate pneumococcal vaccine on carriage and drug resistance of Streptococcus pneumoniae in healthy children attending day-care centers in Lisbon. Pediatr Infect Dis J. 2005;24(3):243-52.

9. Ghaffar F, Barton T, Lozano J, Muniz LS, Hicks P, Gan $\mathrm{V}$, et al. Effect of the 7-valent pneumococcal conjugate vaccine on nasopharyngeal colonization by Streptococcus pneumoniae in the first 2 years of life. Clin Infect Dis. 2004;39(7):930-8.

10. Cullotta AR, Kalter HD, Delgado J, Gilman RH, Facklam RR, Velapatino B, et al. Antimicrobial susceptibilities and serotype distribution of Streptococcus pneumoniae isolates from a low socioeconomic area in Lima. Peru. Clin Diagn Lab Immunol. 2002;9(6):1328-31.

11. Ochoa TJ, Rupa R, Guerra H, Hernandez H, Chaparro E, Tamariz J, et al. Penicillin resistance and serotypes/ serogroups of Streptococcus pneumoniae in nasopharyngeal carrier children younger than 2 years in Lima, Peru. Diagn Microbiol Infect Dis. 2005;52(1):59-64.

12. Perilla M J, Ajello G, Bopp C, Elliot J, Facklam R, Knapp J S, et al. Manual de Laboratorio para la identificación y prueba de susceptibilidad a los antimicrobianos de patógenos bacterianos de importancia para la Salud Pública en el mundo en desarrollo. Haemophilus influenzae, Neisseria meningitidis, Streptococcus pneumoniae, Neisseria gonorrhoeae, Salmonella serotipo Typhi y Vibrio cholerae. Atlanta, Georgia: OMS;.2004, p. 49-67; 269-73.

13. Joshi HH, Gertz RE Jr, da Gloria Carvalho M, Beall BW. Use of silica desiccant packets for specimen storage and transport to evaluate pneumococcal nasopharyngeal carriage among Nepalese children. J Clin Microbiol. 2008;46(9):3175-6.

14. Quintero B, Araque M. Perfil serológico y antibiotipia de cepas de Streptococcus pneumoniae aisladas de portadores nasales pediátricos. Invest Clin. 2006;47(1):17-26.

15. Solórzano-Santos F, Ortiz-Ocampo LA, Miranda-Novales MG, Echániz-Avilés G, Soto-Noguerón A, GuiscafréGallardo H, et al. Serotipos prevalentes de Streptococcus pneumoniae colonizadores de nasofaringe, en niños del Distrito Federal. Salud Publica Mex. 2005;47(4):276-81.

16. Mogdasy MC, Camou T, Fajardo C, Hortal M. Colonizing and invasive strains of Streptococcus pneumoniae in Uruguayan children: type distribution and patterns of antibiotic resistance. Pediatr Infect Dis J. 1992;11(8):648-52.

17. Trucco O, Prado V, Inostroza J, Cabrera E, Teran $\mathrm{R}$, Bravo R, et al. Prevalencia de Streptococcus pneumoniae resistente a penicillina en ninos que asisten a jardines infantiles en Santiago. Rev Chil Pediatr. 1998;67(5):195-9.

18. Kuch A, Sadowy E, Skoczyńska A, Hryniewicz W. First report of Streptococcus pneumoniae serotype 6D isolates from invasive infections. Vaccine. 2010;28(39):6406-7.

19. Jin P, Kong F, Xiao M, Oftadeh S, Zhou F, Liu C, et al. First report of putative Streptococcus pneumoniae serotype $6 \mathrm{D}$ among nasopharyngeal isolates from Fijian children. $J$ Infect Dis. 2009;200(9):1375-80. 
20. Mercado EH, Srinivasan V, Hawkins $P$, Chochua S, Ochoa $T$, Beall B, et al. First report of Streptococcus pneumoniae serotype 6D in South America [letter]. J Clin Microbiol. 2011;49(5):2080-1.

21. Bello González T, Rivera-Olivero IA, Pocaterra L, Spadola E, Araque M, Hermans PW, et al. Estado de portador nasofaríngeo de Streptococcus pneumonia en madres $\mathrm{e}$ hijos de la población indígena Panare del estado Bolívar. Venezuela. Rev Argent Microbiol. 2010;42(1):30-4.

22. Lagos R, MuñozA, San Martin O, Maldonado A, Hormazabal $\mathrm{JC}$, Blackwelder WC, et al. Age- and serotype-specific pediatric invasive pneumococcal disease: insights from systematic surveillance in Santiago, Chile, 1994-2007. J Infect Dis. 2008;198(12):1809-17.

23. Gamboa L, Camou T, Hortal M, Castañeda E; SirevaVigía Working Group. Dissemination of Streptococcus pneumoniae clone Colombia-5-19 in Latin America. J Clin Microbiol. 2002;40(11):3942-50.
24. Pichichero ME, Casey JR.Emergence of a multiresistant serotype 19A pneumococcal strain not included in the 7 -valent conjugate vaccine as an otopathogen in children. JAMA. 2007;298(15):1772-8.

25. Diaz S, Morales S, Gonzalez D, Salazar E. Serotipos de Streptococcus pneumoniae aislados de procesos invasivos, 1999-Abril 2000. Libro de Resúmenes del VII Congreso Peruano de Enfermedades Infecciosas y Tropicales 2001; 30 de agosto al 2 de setiembre. Lima, Perú. Sociedad Peruana de Enfermedades Infecciosas y Tropicales. 2001. p. 30.

Correspondencia: Theresa J. Ochoa

Dirección: Av. Honorio Delgado 430, Lima 31, Perú.

Teléfono: (511) 4823910 / (511) 4823404

Correo electrónico: Theresa.J.Ochoa@uth.tmc.edu

\section{Suscríbase en forma electrónica y gratuita a los contenidos de la Revista Peruana de Medicina Experimental y Salud Pública, ingrese a WWW.ins.gob.pe, seleccione el ícono de la revista y envíenos sus datos.}
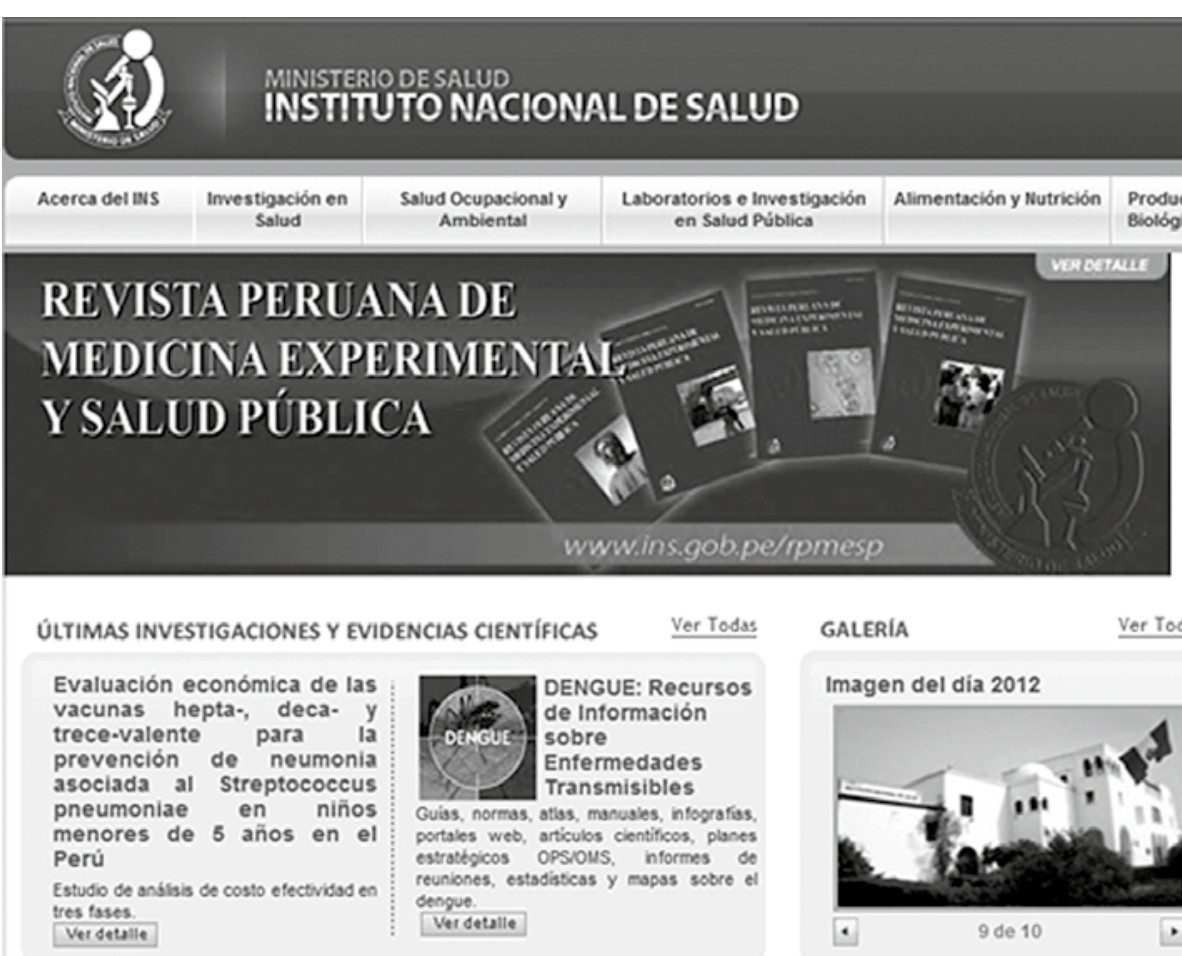

DENGUE: Recursos de Información sobre

Enfermedades Transmisibles Guis, normss, attas, manuales, infografiss. portales web, articulos cientificos, planes estratiogicos OPSIOUS, informes of reuniones, estadiatcos y mapas sobre el dengue. Ver detalle
GALERIA

Ver Todss

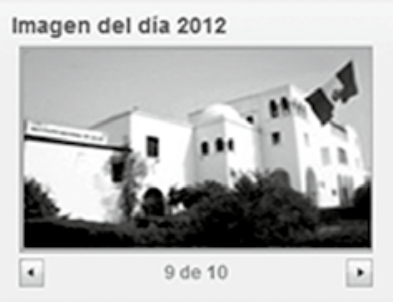

Buscar. B roductos Salud Control de Calided Intercultural de Medicamentos
Q Portal del Transparencia

- Gestión de calidad

6 Directorio instiucional

10. 21 maro cel 2012

A. Agregar a favorios

- Enviar a un amigo

Publicaciones

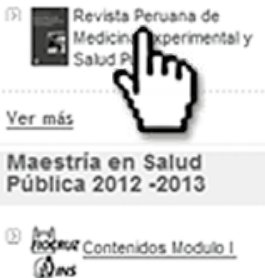

